\title{
Biliary Secretion of Fluid-phase Markers by the Isolated Perfused Rat Liver Role of Transcellular Vesicular Transport
}

John R. Lake, Vojtech Licko, Rebecca W. Van Dyke, and Bruce F. Scharschmidt

Department of Medicine and Liver Center, University of California, San Francisco, California 94143

\begin{abstract}
In these studies, we have used several approaches to systematically explore the contribution of transcellular vesicular transport (transcytosis) to the blood-to-bile movement of inert fluid-phase markers of widely varying molecular weight. First, under steady-state conditions, the perfused rat liver secreted even large markers in appreciable amounts. The bile-to-plasma $(B / P)$ ratio of these different markers, including microperoxidase $(B / P$ ratio $=0.06 ; \mathrm{mol} w t=1,879)$, inulin $(B / P$ ratio $=0.09$, mol wt $=5,000)$, horseradish peroxidase $(B / P$ ratio $=0.04, \mathrm{~mol} w \mathrm{wt}=40,000)$, and dextran $(\mathrm{B} / \mathrm{P}$ ratio $=0.09$, mol $w t=70,000)$, exhibited no clear ordering based on size alone, and when dextrans of two different sizes $(40,000$ and 70,000 mol wt) were studied simultaneously, the relative amounts of the two dextran species in bile were the same as in perfusate. Taurocholate administration produced a $71 \%$ increase in bile flow but little or no (0-20\%) increase in the output of horseradish peroxidase, microperoxidase, inulin, and dextran. Second, under nonsteady-state conditions in which the appearance in or disappearance from bile of selected markers was studied after their abrupt addition to or removal from perfusate, erythritol reached a $B / P$ ratio of 1 within $2 \mathrm{~min}$. Microperoxidase and dextran appeared in bile only after a lag period of $\sim 12$ min and then slowly approached maximal values, whereas sucrose exhibited kinetically intermediate behavior. A similar pattern was observed after removal of $>95 \%$ of the marker from the perfusate. Erythritol rapidly reapproached a $B / P$ ratio of 1 , whereas the $B / P$ ratio for sucrose, dextran, and microperoxidase fell much more slowly and exceeded 1 for a full $30 \mathrm{~min}$ after perfusate washout. Finally, electron microscopy and fluorescence microscopy of cultured hepatocytes demonstrated the presence of horseradish peroxidase and fluoresceindextran, respectively, in intracellular vesicles, and fractionation of perfused liver homogenates revealed that at least $35-50 \%$ of sucrose, inulin, and dextran was associated with subcellular organelles.
\end{abstract}

Collectively, these observations are most compatible with a transcytosis pathway that contributes minimally to the secretion of erythritol, but accounts for a substantial fraction of sucrose secretion and virtually all (>95\%) of the blood-to-bile

Portions of this work were presented at the annual meeting of the American Gastroenterological Association, May 1984, and were published in abstract form, 1984, Gastroenterology. 86:1237.

Address reprint requests and correspondence to Dr. Scharschmidt, Gastrointestinal Research Unit, 1120 HSW, University of California, San Francisco, CA 94143.

Received for publication 31 December 1984.

J. Clin. Invest.

(c) The American Society for Clinical Investigation, Inc. 0021-9738/85/08/0667/09 \$1.00

Volume 76, August 1985, 676-684 transport of microperoxidase and larger markers. These findings have important implications with respect to current concepts of canalicular bile formation as well as with respect to the conventional use of solutes such as sucrose as markers of canalicular or paracellular pathway permeability.

\section{Introduction}

Vesicles are involved in the uptake and intracellular transport of a variety of macromolecules such as low density lipoproteins, asialoglycoproteins, and immunoglobulin $\mathrm{A}$, which enter hepatocytes via receptor-mediated endocytosis (1-3). Certain of these substances, such as asialoglycoproteins, are transported largely to lysosomes where they are catabolized $(1,4)$, while only a small proportion appears intact in bile (4). Ligands such as immunoglobulin $A$ appear to be transported directly to bile without passing through the lysosomal compartment (3). Horseradish peroxidase, a marker of fluid phase endocytosis, has also been found in bile after intravenous injection $(3,5)$. Despite these observations, relatively little is known regarding the role of transcellular vesicular transport in canalicular bile formation.

The route by which fluid and solutes enter bile, while of considerable interest in its own right, is a particularly important consideration when studying the blood-to-bile transport of inert markers such as erythritol, sucrose, and inulin (6). Despite acknowledged uncertainty regarding the locus (canalicular versus ductular) and/or mechanism of their entry into bile (6-10), the equations currently used to describe the movement of these widely used markers are based on movement via pore-restricted diffusion and bulk flow (9-12), and the movement of sucrose or larger markers is commonly assumed to occur via a paracellular pathway. Indeed, under conditions of altered bile flow, changes in the bile-to-plasma $(B / P)^{1}$ ratio of inert markers such as sucrose are often described in terms of changes in the osmotic permeability or sieving coefficient and interpreted as altered permeability or "leakiness" of the hepatocyte tight junctions (13-15).

Therefore, the purpose of these studies was to systematically explore the possible contribution of transcellular vesicular transport to the blood-to-bile transport of inert fluid phase markers. Our experimental strategy has been to study the perfusate-to-bile transport of markers of widely varying molecular weights under steady-state as well as nonsteady-state conditions and to look directly for the presence of these markers in intracellular vesicles.

\section{Methods}

\section{Materials and reagents}

Fluorescein isothiocyanate dextran (70,000 average mol wt), horseradish peroxidase type II, microperoxidase (MP-11), dextran (81,600 average

1. Abbreviations used in this paper: $\mathrm{B} / \mathrm{P}$, bile-to-plasma. 
mol wt), inulin (5,000 mol wt), and taurocholate were obtained from Sigma Chemical Co., St. Louis, MO. 6-Carboxyfluorescein diacetate was obtained from Molecular Probes, Inc., Junction City, OR. $\left[{ }^{14} \mathrm{C}\right]-$ carboxyl-dextran $(70,000$ average $\mathrm{mol} \mathrm{wt}),\left[{ }^{14} \mathrm{C}\right]$ sucrose, $\left[{ }^{14} \mathrm{C}\right]$ methoxyinulin (5,000 average mol wt) were obtained from New England Nuclear, Boston, MA. $\left[{ }^{3} \mathrm{H}\right]$ dextran $(70,000$ average mol wt), $\left[{ }^{3} \mathrm{H}\right]$ sucrose, and $\left[{ }^{14} \mathrm{C}\right]$ erythritol were obtained from Amersham Corp., Arlington Heights, IL. $\left[{ }^{3} \mathrm{H}\right]$ dextran $(40,000$ average $\mathrm{mol} \mathrm{wt})$ was kindly prepared by Dr. Chin-Tzu Peng at the Lawrence Berkeley Laboratory using a microwave discharge activation technique with ${ }^{3} \mathrm{H}$ gas. Fluosol43 was obtained from Alpha Therapeutics, Los Angeles, CA.

\section{Analytical and preparative techniques}

The fluorescein isothiocyanate dextran used in these studies was dissolved in $0.1 \mathrm{M}$ phosphate-buffered saline (PBS) and separated from free fluorescein and other low-molecular-weight fluoresceinated contaminants by passage over a Sephadex G-75 column $(0.9 \mathrm{~cm} \times 25$ $\mathrm{cm})$. The void volume fraction was dialyzed against distilled water overnight at $4^{\circ} \mathrm{C}$, lyophilized, and stored dessicated at $4^{\circ} \mathrm{C}$ until used. Radiolabeled dextran was prepared for use in a similar fashion.

Horseradish peroxidase and microperoxidase in bile and perfusate were measured spectrophotometrically as the rate of oxidation of 4 aminoantipyrine at $510 \mathrm{~nm}$ as previously described (16). Radioactivity was measured by liquid scintillation counting in Aquasol (New England Nuclear) using external or internal standardization for quench correction.

\section{Experimental systems}

Isolated perfused rat liver. The surgical techniques, design, and operation of the perfused rat liver apparatus used in these studies have been described in detail previously (17-19). A constant perfusion rate of 25 $\mathrm{ml} / \mathrm{min}$ was maintained in all studies and net portal perfusion pressure was $<5 \mathrm{~cm} \mathrm{H}_{2} \mathrm{O}$ during all experiments. The $\mathrm{pH}$ was continuously monitored and maintained between 7.35 and 7.45 by either slight adjustments in the $\mathrm{pCO}_{2}$ or by the addition of small amounts of a concentrated bicarbonate solution. This system using perfusate containing 20\% (vol/vol) fluorocarbon emulsion (Fluosol-43) as an oxygen carrier has been extensively validated. Viability of the perfused liver for perfusions up to $3 \mathrm{~h}$ has been shown by normal appearance by electron microscopy, stable perfusate lactate dehydrogenase and transaminase activity, and normal hepatic oxygen consumption (17-19).

Two perfusion protocols were utilized for these studies. The first protocol (steady-state protocol) lasted a total of $150 \mathrm{~min}$. The first 90 min involved continuous perfusion in a recirculating fashion with bile acid-free perfusate containing the various markers under study. At 90 min, $2.5 \mu \mathrm{mol}$ of taurocholate was introduced into the reservoir as a bolus to yield a perfusate concentration of $\sim 40 \mu \mathrm{M}$. This was followed by a continuous infusion of $90 \mathrm{nmol} / \mathrm{g} \cdot \mathrm{liver} \cdot \mathrm{min}$ for the final $60 \mathrm{~min}$ (18). Bile was collected throughout the perfusion in 10-min aliquots in pretared polypropylene containers (Bio-Rad Laboratories, Richmond, CA), and volume was determined gravimetrically, assuming a density of $1 \mathrm{~g} / \mathrm{ml}$. Marker concentration in perfusate was also determined at 10 - to 15 -min intervals by measuring radioactivity in an aliquot of perfusate and correcting for the volume of the fluorocarbon beads (average $\mathbf{~} 20 \%$ ).

The second protocol (nonsteady-state protocol) was designed to permit a kinetic analysis of the rate of movement of the various markers between perfusate and bile, and lasted a total of $150 \mathrm{~min}$. The first $30 \mathrm{~min}$ represented a stabilization period in which the liver was perfused with bile acid-free and marker-free fluorocarbon-containing perfusate. At $30 \mathrm{~min}$, a new perfusate containing appropriate marker(s) was abruptly introduced into the system as previously described (1719) so as to produce a "square-wave" increase in marker concentration. At 75 min, marker was flushed from the system by a 2-min period of nonrecirculating perfusion with marker-free Krebs-Hensleit buffer followed by $43 \mathrm{~min}$ of recirculating perfusion with marker-free perfusate in a fashion similar to that previously employed for ion substitution experiments (17-19). Finally, to assess the possible effect of bile acids on washout of residual liver-associated marker, a $2.5-\mu \mathrm{mol}$ bolus of taurocholate was introduced at $120 \mathrm{~min}$ followed by taurocholate infusion for the final $30 \mathrm{~min}$ (18). Marker concentration in perfusate was determined every 10 to $15 \mathrm{~min}$ and bile was collected in calibrated capillary tubes varying in volume from 10-50 $\mu \mathrm{l}$ (Drummond Scientific Co., Broomall, PA). The elapsed time for each sample collection was recorded, and marker concentration in the sample was determined. This approach, using volume-based collections, permitted determination of bile flow and the $\mathrm{B} / \mathrm{P}$ ratio of markers for time increments as short as $30 \mathrm{~s}$.

For both protocols, $2-5 \mu \mathrm{Ci}$ of radiolabeled marker was used for each perfusion in the presence of a variable amount of nonradiolabeled marker. For each marker, biliary clearance of radiolabel and the $B / P$ ratio were constant over a $2-\log$ perfusate concentration range, indicating a lack of saturable transport or binding. Horseradish peroxidase and microperoxidase were present in perfusate in concentrations of $0.2-1$ $\mathrm{mg} / \mathrm{ml}$ and $0.2-0.5 \mathrm{mg} / \mathrm{ml}$, respectively, with biliary output of each remaining constant over these perfusate concentration ranges. Binding of the various markers to fluorocarbon beads, determined by centrifugation of perfusate, was absent or minimal $(<10 \%)$, and the volume of beads $(20 \%)$ has been corrected for in all calculations of marker concentration in perfusate.

Sizing of dextrans. Radiolabeled dextrans in bile and perfusate were analyzed using methods previously described (20). Dextrans were separated according to size on a Sephacryl S-200 $(1.6 \mathrm{~cm} \times 95 \mathrm{~cm})$ column using $0.3 \%$ saline as the eluting buffer. $2-\mathrm{ml}$ fractions were collected using an automatic fraction collector (LKB) and analyzed for radioactivity. Einstein-Stokes radii for eluted dextrans were calculated from the fractional volume available to the solute $\left(\mathrm{K}_{\mathrm{av}}\right)(21)$ determined from the equation: $K_{\mathrm{av}}=\left(V_{\mathrm{c}}-V_{0}\right) /\left(V_{\mathrm{t}}-V_{0}\right)$, where $V_{0}$ is the void volume determined with blue dextran, $V_{\mathrm{c}}$ is the elution volume of the solute, and $V_{\mathrm{t}}$ is the total volume of the column. To determine peak areas in studies employing more than our dextran species, multiple Gaussian distributions were fitted to the dextran elution profile by a nonlinear least squares procedure and the areas under the curves estimated for perfusate and bile.

\section{Subcellular fractionation (Fig. 1)}

To quantitate the amount of radiolabeled marker contained in subcellular organelles, livers were perfused for $60 \mathrm{~min}$ with a recirculating fluorocarbon perfusate containing various markers in radiolabeled form. At 60 min the liver was flushed with $20 \mathrm{~cm}^{3}$ of ice-cold KrebsHenseleit solution. Two pieces of liver weighing 3-5 $\mathrm{g}$ each were rapidly cut off, weighed, minced, and homogenized (H1) using 20 strokes in a loose-fitting Dounce containing $5 \mathrm{~cm}^{3}$ of $0.01 \mathrm{M} \mathrm{PBS} / \mathrm{g}$ liver. The homogenate was centrifuged at $1,085 \mathrm{~g}$ in a Sorvall SS-34 rotor for $5 \mathrm{~min}$ to pellet (P1) intact cells and cell clumps; the absence of intact cells in the supernatant (S1) was confirmed by bright-field microscopy using a Zeiss light microscope (Carl Zeiss, Inc., Thornwood, NY) at a magnification of $400 \times$. The supernatant (S1) was divided into two equal parts with one part rehomogenized (H2) with 10 strokes in a loose dounce containing an equal volume of $0.01 \mathrm{M}$ PBS and the remaining part rehomogenized $(\mathrm{H} 2)$ in a similar fashion, except that the $0.01 \mathrm{M}$ PBS contained $1 \%$ Triton X-100. These two homogenates were each centrifuged at $100,000 \mathrm{~g}$ for $60 \mathrm{~min}$ in a 42.1 fixed-angle rotor (Beckman Instruments, Inc., Fullerton, $\mathrm{CA}$ ). The pellets (P2 and $\left.\mathrm{P}^{\prime}\right)$ and aliquots of homogenate $\left(\mathrm{H} 1, \mathrm{H} 2\right.$, and $\mathrm{H}^{\prime}$ ) were solubilized using Soluene 350. These, as well as aliquots of the supernatant (S1, S2, and S2'), were decolorized using hydrogen peroxide and isopropyl alcohol and were placed in Dimilume (Packard Instruments Corp., Downers Grove, IL) for scintillation counting. The original pellets (P1) were also rehomogenized (H3), and subjected again to low speed $(1,085$ g) centrifugation to pellet residual intact cells (P3). The supernatant (S3) was rehomogenized in the presence $\left(\mathrm{H}^{\prime}\right)$ or absence $(\mathrm{H} 4)$ of $1 \%$ Triton X-100, recentrifuged, fractionated, and counted in precisely the same fashion as described above for the original supernatant both in the absence $(\mathrm{H} 4 \rightarrow \mathrm{S} 4, \mathrm{P} 4)$ and presence $\left(\mathrm{H}^{\prime} \rightarrow \mathrm{S} 4^{\prime}, \mathrm{P}^{\prime}\right)$ of detergent. To exclude nonspecific adherence of marker to sedimenting organelles, control studies were conducted by adding radiolabeled marker to tissue 


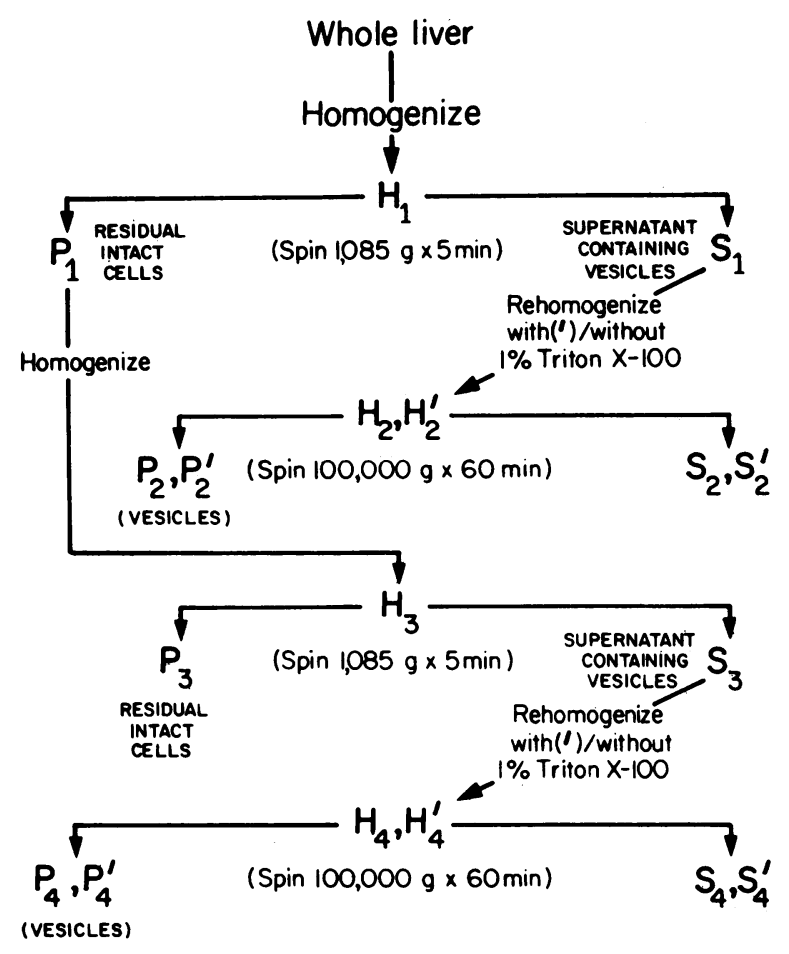

Figure 1. Schematic representation of subcellular fractionation protocol.

excised from livers perfused for 60 min with marker-free perfusate during the initial homogenization (H1). This tissue was then fractionated and processed as described above.

In all studies, isotope recovery and distribution among the various fractions was determined. Of particular interest was the distribution of isotope present in homogenate $\left(\mathrm{H} 2, \mathrm{H} 2^{\prime} ; \mathrm{H} 4, \mathrm{H}^{\prime}\right)$ between the resulting pellets and supernatants in the presence $\left(\mathrm{P2}^{\prime}, \mathrm{S2}^{\prime} ; \mathrm{P4}^{\prime}, \mathrm{S4}^{\prime}\right)$ and absence (P2, S2; P4, S4) of detergent.

Electron microscopy. Livers were perfused for $50 \mathrm{~min}$ with a fluorocarbon perfusate containing $1 \mathrm{mg} / \mathrm{ml}$ horseradish peroxidase and perfusion-fixed via the portal vein for 2 min with $25 \%$ glutaraldehyde and $0.8 \%$ paraformaldehyde in $0.2 \mathrm{M}$ sodium-bicarbonate buffer. Liver tissue was then immersed in the same fixative for an additional $2 \mathrm{~h}$ and chopped into 40-nm sections using a Smith-Farquhar chopper. After an overnight wash in $0.2 \mathrm{M}$ sodium-bicarbonate buffer, sections were tested for peroxidase activity using the diaminobenzidine technique of Graham and Karnovsky (22). To evaluate endogenous peroxidase, control livers perfused with horseradish peroxidase-free perfusate were also examined. Diaminobenzidine reaction controls, in which hydrogen peroxide was omitted from the medium, were also performed.

Tissues were then osmicated in $1 \%$ osmium tetroxide containing $1.5 \% \mathrm{KCN}$ for $90 \mathrm{~min}$, washed in $0.2 \mathrm{M}$ sodium-bicarbonate for 10 min, dehydrated in ethanol, and embedded in Epon. Sections were stained with lead citrate and examined in an electron microscope (300; Philips Electronic Instruments, Inc., Mahwah, NJ).

Cultured cells. Rat hepatocytes were prepared by collagenase perfusion, plated on plastic dishes as previously described, and maintained in modified 1990R medium supplemented with amino acids, insulin, and corticosteroid for $48 \mathrm{~h}$ before use (23). Cell viability was assessed by release of lactate dehydrogenase as previously described (24). The 48-h-old cultures were incubated for $10 \mathrm{~min}$ to $16 \mathrm{~h}$ in media containing $25 \mathrm{mg} / \mathrm{ml}$ fluorescein-isothiocyanate dextran (70,000 average mol wt). The supernatant medium was then aspirated with a Pasteur pipette, washed with buffer, and incubated in fluorochrome-free medium for $10 \mathrm{~min}$ to $3 \mathrm{~h}$ before visualization. Cells were visualized by transmission and fluorescence illumination using an Olympus fluorescent microscope
(Olympus Corporation of America, New Hyde Park, NY). For visualization of cells exposed to carboxyfluorescein, 48-h cultures were incubated in medium containing 6-carboxyfluorescein diacetate (45 $\mu \mathrm{g} / \mathrm{ml}$ ) for $10 \mathrm{~min}$ and visualized as above. Photographs were taken with an Olympus C-35 camera (Olympus Corporation of America) using ASA 400 Ektachrome film (Eastman Kodak Co., Rochester, NY).

Mathematical analysis nonsteady-state studies. A total of 20 nonsteady-state experiments was performed using the various fluid phase markers (erythritol, 5; sucrose, 5; microperoxidase, 2; horseradish peroxidase, 3; dextran, 5), and for each study the bile-to-perfusate concentration ratio was calculated for 20-32 time points spaced at 30$s$ to 2-min intervals. The mean bile-to-perfusate concentration ratios for each marker at each 1-min time interval were determined by moving averages of the order $N+1$. These mean data were analyzed with a Hewlett-Packard desktop computer system (HP98258/9872A; Hewlett-Packard Co., Palo Alto, CA) by a nonlinear least-squares procedure utilizing the Euler method for numerical solution of the differential equations. Various kinetic models were tested for each solute, including direct transfer from blood-to-bile without an interposed compartment, or transit through one or more interposed delay compartments. The equations were iteratively augmented to determine the simplest system (combination of pathways) that satisfactorily described the movement of all solutes individually and collectively. The best-fit equations were determined to be those that optimized (minimized) the sum of squares $F$ values as well as parameter uncertainties. In the initial analyses, all parameters were permitted to vary freely. In the final analysis, certain parameters (see Results, Fig. 3, Table II) were fixed, and the remaining parameters were varied until the simplest overall system was determined.

\section{Results}

Secretion of markers by perfused liver under steady-state conditions. All solutes had reached maximal values in bile by 45 min, with less than a $15 \%$ coefficient of variation of the biliary concentration of all these solutes between 50 and $90 \mathrm{~min}$. The results of these studies, which are summarized in Table $I$, indicate that even the largest of the solutes (i.e., 70,000 mol wt dextran, which achieved a B/P ratio of 0.09 ) appeared in bile in significant quantities. Moreover, the dextran present in

Table I. Steady-state Bile-to-Perfusate Ratio in the Absence of Bile Acids and During Taurocholate Infusion for Various Fluid-phase Markers

\begin{tabular}{|c|c|c|}
\hline \multirow[b]{2}{*}{ Marker } & \multicolumn{2}{|c|}{ Steady-state bile-to-perfusate ratio* } \\
\hline & $\begin{array}{l}\text { Absence of } \\
\text { bile acids }\end{array}$ & $\begin{array}{l}\text { Taurocholate } \\
\text { infusion }\end{array}$ \\
\hline Erythritol (122 mol wt) & $1.0 \pm 0.03$ & $1.0 \pm 0.04$ \\
\hline Sucrose (342 mol wt) & $0.21 \pm 0.01$ & $0.16 \pm 0.01$ \\
\hline \multicolumn{3}{|l|}{ Microperoxidase } \\
\hline$(1,879 \mathrm{~mol} \mathrm{wt})$ & $0.06 \pm 0.01$ & $0.04 \pm 0.01$ \\
\hline \multicolumn{3}{|l|}{ Methoxyinulin } \\
\hline$(5,000$ average $\mathrm{mol} w t)$ & $0.09 \pm 0.01$ & $0.06 \pm 0.01$ \\
\hline \multicolumn{3}{|l|}{ Horseradish peroxidase } \\
\hline$(40,000 \mathrm{~mol} \mathrm{wt})$ & $0.04 \pm 0.04$ & $0.03 \pm 0.02$ \\
\hline \multicolumn{3}{|l|}{ Dextran } \\
\hline$(70,000$ average mol wt) & $0.09 \pm 0.03$ & $0.05 \pm 0.02$ \\
\hline
\end{tabular}

* Each value represents the mean \pm SD derived from 4-6 perfusions for each marker. Bile flow averaged $1.32 \mu \mathrm{l} / \mathrm{min} \cdot \mathrm{g}$ during taurocholate infusion (120-150 min). 
both bile and perfusate was shown chromatographically to consist almost exclusively ( $>95 \%$ ) of high-molecular-weight (Einstein-Stokes radius $\geq 43 \AA$ ) material. The results in Table I also indicate that solutes with very different molecular weights exhibited similar B/P ratios. Indeed, over the molecularweight range of 1,800 to 70,000 , there was no apparent ordering of the biliary secretion of these solutes based on size. In addition, when chromatographically prepared dextrans of two different sizes $(70,000$ and $40,000 \mathrm{~mol}$ wt) were added simultaneously to perfusate, the chromatographic profile of biliary dextran was similar to that of perfusate dextran, and the relative amounts of the two dextrans, determined chromatographically, in bile $(38.3 \%, 70,000 \mathrm{~mol} w \mathrm{wt} 61.7 \%, 40,000$ $\mathrm{mol} \mathrm{wt}$ ) and perfusate $(39.5 \%, 70,000 \mathrm{~mol} \mathrm{wt} ; 60.5 \% 40,000$ mol wt) were virtually identical.

The addition of taurocholate to the perfusate produced a $71 \%$ increase in bile flow (18), and a new steady-state $B / P$ ratio was reached by $120 \mathrm{~min}(30 \mathrm{~min}$ after beginning taurocholate infusion) with the coefficient of variation of the biliary concentration of the markers being $<20 \%$ thereafter. Except for erythritol, the output of the various markers either remained unchanged (dextran) or increased much less than the increase in bile flow (inulin, 15.7\%; horseradish peroxidase, $18.7 \%$; microperoxidase, $16.2 \%$; sucrose, $43.6 \%$ ), resulting in a fall in the bile-to-plasma ratios. These data are also summarized in Table I.

Collectively, these observations indicate that large fluid phase markers enter bile in appreciable quantities via a pathway that is minimally affected by taurocholate. Based on the results with inulin, microperoxidase, horseradish peroxidase, and dextrans of two different molecular weights, this pathway exhibits little selectivity based on size.

Secretion of markers by perfused liver under nonsteadystate conditions. To better define the relative rates at which the various markers move from perfusate to bile, a series of studies was conducted (using the protocol described in Methods) in which markers were abruptly introduced into and then removed from perfusate with frequent volume-based bile collection. The results of these studies for erythritol, sucrose, and dextran are illustrated in Fig. 2. After its abrupt addition to perfusate, erythritol reached a concentration in bile equal to that in plasma within $\sim 2 \mathrm{~min}$. Indeed, the movement of erythritol was so rapid that even the sampling technique employed in these studies was not adequate to fully define it. Note that this rapid appearance of erythritol into bile does not take into account any correction for biliary deadspace. The deadspace attributable to the biliary collection cannula (i.d. $=0.28 \mathrm{~mm} ; 12 \mathrm{~cm}$ length) was $7.4 \mu \mathrm{l}$. When this was added to even the lowest conventional estimate of hepatic biliary deadspace of $2.3 \mu \mathrm{l} / \mathrm{g}$ liver (25), the calculated time for deadspace transit of $\sim 2$ min equaled or exceeded the observed lag time before erythritol appearance in bile (Fig. 2). We therefore chose to use no correction for deadspace, rather than an arbitrary and possibly incorrect one. It is also interesting in this regard that the conventional estimates of biliary deadspace are derived from the use of markers (taurocholate and sulfobromophthalein) that pass through, rather than between, hepatocytes $(25,26)$. The lesser value for deadspace based on erythritol movement thus raises interesting questions with respect to the route of erythritol movement (see below) and/ or the mechanism of taurocholate-induced choleresis, upon which one current estimate rests (25).

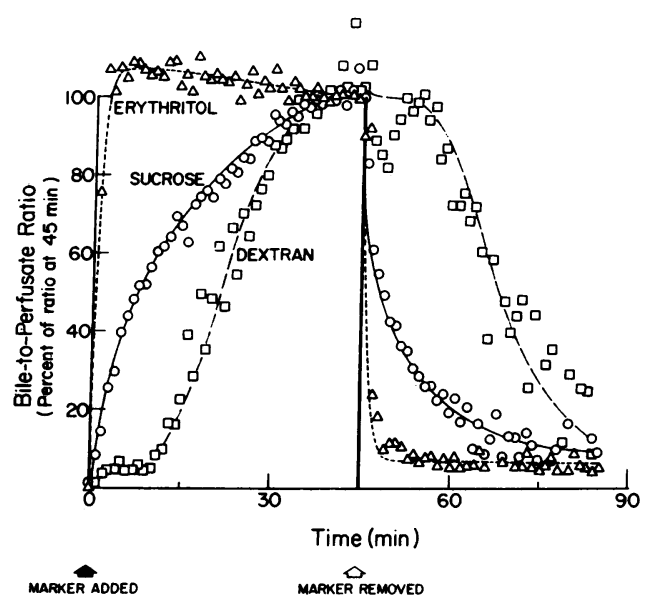

Figure 2. Appearance/disappearance curves for erythritol (120 mol $w t)$, sucrose (342 mol wt), and dextran (70,000 average mol wt) after their abrupt addition to and removal from perfusate.

In contrast to erythritol, dextran appeared in bile in appreciable quantities only after a lag of $\sim 12 \mathrm{~min}$, after which it gradually approached a mean bile-to-plasma ratio of 0.09 . Sucrose approached a mean bile-to-plasma ratio of 0.21 at an intermediate rate between that of erythritol and sucrose.

After their abrupt removal from perfusate at $75 \mathrm{~min}(95 \%$ complete washout), the rates at which erythritol, sucrose, and dextran disappeared from bile mirrored their rates of appearance as described above (Fig. 2). Note that the absolute bile-toplasma ratio of sucrose and dextran exceeded 1 for $>30$ min after their washout from perfusate, a time period much greater than that $(5 \mathrm{~min})$ necessary to clear even the largest estimate of biliary deadspace (26). This pattern, which contrasts sharply with that of erythritol (Fig. 2), has important implications with respect to the routes of movement of these markers.

Additional studies performed with microperoxidase ( $n$ $=2$, not shown) and horseradish peroxidase $(n=4$, not shown) indicated that these markers exhibited a pattern of appearance in/disappearance from bile similar to that of dextran, differing only in that a lower maximal bile-to-plasma ratio (mean values, 0.06 and 0.04 , respectively) was reached by $75 \mathrm{~min}$.

As with the steady-state studies, the addition of taurocholate (bolus plus infusion) to the perfusate beginning at $120 \mathrm{~min}$ produced the expected choleresis but did not appreciably increase the biliary output and/or biliary concentration of any of the markers except sucrose and erythritol.

Kinetic analysis of marker secretion under nonsteady-state conditions. Several conclusions may be drawn by simple inspection of the data depicted in Fig. 2. Most importantly, the lag exhibited by dextran (also microperoxidase and horseradish peroxidase) is incompatible with a model of movement from perfusate to bile via a pathway described by a single rate constant; rather, this lag requires interposed delay compartments. Erythritol movement, in contrast, was exceedingly rapid and was compatible with a transport pathway that can be adequately described kinetically by a single rate constant. It is also apparent that this exceedingly rapid movement precludes any detailed kinetic characterization of the pathway by which erythritol entered bile. Finally, simple inspection indicates that sucrose movement occurs via a pathway(s) that 
is kinetically intermediate between those of erythritol and dextran.

Detailed mathematical analyses of perfusate-to-bile movement of the various markers was restricted to the wash-in phase of the nonsteady-state studies. Fig. 3 depicts the simplest model, which described the movement of all solutes, individually and collectively; i.e., movement of each solute was satisfactorily described by one or some combination of the pathways depicted.

Table II summarizes the best estimates for each parameter as well as its corresponding uncertainty. The absolute value of these parameters was determined for each solute, both from the pattern of approach to steady-state (Fig. 2) and the absolute steady-state values (Table I).

Demonstration of fluid phase markers in intracellular vesicles. Several approaches were used to study the localization of various markers in liver tissue. First, using the liver fractionation technique described in Methods (Fig. 1), 35-51\% of sucrose, dextran, and inulin present in the $\mathrm{H} 2$ homogenate was found in the P2 pellet containing subcellular organelles in the absence of Triton, as compared with only $7 \%$ of erythritol (Table III). In the presence of Triton, nearly all of the marker present in homogenate $\mathrm{H}^{\prime}{ }^{\prime}$ was released into the supernatant S2' (Table III). When the P1 pellet (which contained an average of $37 \%$ of the radioactivity present in the $\mathrm{H} 1$ homogenate) was itself rehomogenized $\left(\mathrm{H} 4, \mathrm{H}^{\prime}\right)$ and fractionated as described in Methods, the results with respect to distribution of marker between S4, S4' and P4, P4' were identical to those described above, and the results, therefore, have been pooled for presen-

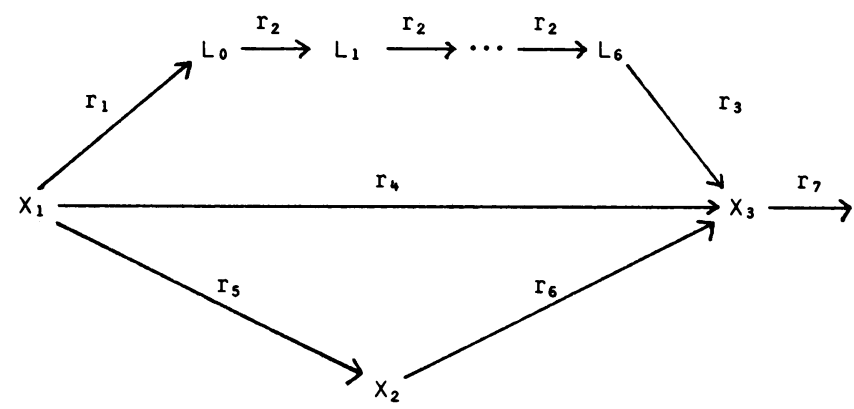

Figure 3. Proposed model for movement of the various solutes from perfusate ( $X_{1}$ compartment) to canaliculus ( $X_{3}$ compartment). Based on the other findings reported in the present manuscript, compartments $\mathrm{L}_{0}$ through $\mathrm{L}_{6}$ are tentatively identified as intracellular vesicles. Rate constant $r_{7}$ described movement of solute from the bile canaliculus to the collection tube. The proposed model is based on an operationally defined system that describes the time course of the appearance of the various markers in bile. The basic characteristics of the bile appearance curve dictate which pathways are to be considered. As summarized in Table II, erythritol traveled exclusively via the direct $\left(r_{4}\right.$, zero-delay) pathway, whereas dextran traveled almost exclusively via the multiple delay $\left(r_{1}, r_{2}, r_{3}\right.$, tentatively transcytosis) pathway. Sucrose movement was best described as a combination of the transcytosis pathway, zero-delay pathway, and a third $\left(r_{5}, r_{6}\right)$ pathway involving only a single delay compartment $\left(\mathrm{X}_{2}\right)$. Inclusion of this third pathway involving a single delay compartment dramatically improved the overall fit of the curve as compared with the direct $\left(r_{4}\right)$ and transcytosis $\left(r_{1}, r_{2}, r_{3}\right)$ pathways alone or in combination. Very little sucrose movement occurred via the direct pathway. Microperoxidase, like dextran, traveled exclusively via the transcytosis pathway. tation in Table III. In contrast, when markers were simply added to perfused/homogenized liver $(\mathrm{H} 1),<5 \%$ was recovered in the P2 pellet. Since some loss of marker from vesicles very likely occurred during homogenization, which may have disrupted some vesicles, or during the fractionation procedure, which required $\sim 2 \mathrm{~h}$, it is likely that these data (Table III) represent a minimal estimate of the percentage of marker present in subcellular compartments.

Second, horseradish peroxidase was shown by electron microscopy to be present within intracellular vesicles (Fig. 4). Finally, exposure of hepatocytes to fluoresceinated dextran and visualization by fluorescence microscopy revealed fluorescence only in distinctly vesicular-like structures (Fig. 5). This contrasted sharply to the diffuse cellular fluorescence which was observed after exposure of the cells to 6-carboxyfluorescein diacetate, which is known to distribute diffusely in cytoplasm (Fig. 5) (27).

\section{Discussion}

The purpose of these studies was to examine the possible role of transcellular vesicular transport (transcytosis) in the bloodto-bile transport of a variety of inert, fluid-phase markers; that is, markers that are not known to bind to specific receptors or carriers on the hepatocyte surface membrane. While the possible contribution of such a pathway to biliary secretion has been previously considered and preliminary evidence consistent with its existence has been reported $(14,28)$, we are unaware of any previous systematic study of this issue. Our overall strategy involved the use of several different approaches, and the results obtained with each will be discussed in turn.

Studies examining the secretion of markers by the perfused rat liver under steady-state conditions yielded several important findings. First, even markers such as 70,000 mol wt dextran, which are generally believed to be too large to pass readily through tight junctions, were secreted into bile in appreciable quantities (Table I). Second, there was no clear ordering with respect to the rate of secretion based on size. Indeed, given the similar rates of secretion of like substances of differing size (microperoxidase and horseradish peroxidase; dextrans with bimodal size distribution), the pathway exhibits no evidence of selectivity based on size. This conclusion must of course be considered tentative until a still wider range of solutes of differing size are systematically studied. It must be pointed out that the similar rates of secretion of different-sized markers such as sucrose and inulin have previously been noted by Bradley and Merz (29). However, these investigators interpreted this finding as evidence for the existence of functionally large (5-10 $\AA$ ) pores in the paracellular pathway, and the secretion of intermediate (e.g., microperoxidase) or larger solutes (dextran, horseradish peroxidase) was not studied. Finally, the present studies indicate that taurocholate produces little or no increase $(0-20 \%)$ in the transport to bile of markers greater than 1,800 mol wt.

We also studied the appearance in/disappearance from bile of selected markers under nonsteady-state conditions. This novel approach differs from the steady-state type analyses described above or reported by previous investigators $(9,10$, $12,15)$ and yielded additional important information. The single most important finding was the clear-cut time lag that occurred before dextran appeared in bile in contrast to erythritol or sucrose. This behavior was exhibited also by microperoxidase 
Table II. Estimated Rate Constants for the Various Solutes $\left(\mathrm{min}^{-1}\right)^{*}$

\begin{tabular}{|c|c|c|c|c|}
\hline & $r_{1}$ & $r_{s}$ & $\mathrm{r}_{6}$ & $\mathbf{r}_{4}$ \\
\hline Erythritol & $-\ddagger$ & - & - & $\begin{array}{r}6.66\left(10^{-4}\right) \\
\pm 5.67\left(10^{-8}\right)\end{array}$ \\
\hline Sucrose & $\begin{array}{r}6.64\left(10^{-5}\right) \\
\pm 1.37\left(10^{-7}\right)\end{array}$ & $\begin{array}{r}1.18\left(10^{-4}\right) \\
\pm 1.61\left(10^{-7}\right)\end{array}$ & $\begin{array}{r}1.70\left(10^{-1}\right) \\
\pm 2.50\left(10^{-2}\right)\end{array}$ & $\begin{array}{r}1.12\left(10^{-5}\right) \\
\pm 5.90\left(10^{-8}\right)\end{array}$ \\
\hline Dextran $\left(r_{3}=\infty\right)$ & $\begin{array}{r}1.12\left(10^{-4}\right) \\
\pm 6.04\left(10^{-8}\right)\end{array}$ & - & - & $\begin{array}{r}2.05\left(10^{-6}\right) \\
\pm 5.41\left(10^{-9}\right)\end{array}$ \\
\hline Microperoxidase & $\begin{array}{r}2.45\left(10^{-5}\right) \\
\pm 2.60\left(10^{-8}\right)\end{array}$ & - & - & - \\
\hline $\begin{array}{l}\text { Horseradish peroxidase } \\
\qquad\left(r_{3}=\infty\right)\end{array}$ & $\begin{array}{r}4.07\left(10^{-6}\right) \\
\pm 4.30\left(10^{-8}\right)\end{array}$ & $\begin{array}{r}1.95\left(10^{-5}\right) \\
\pm 1.85\left(10^{-6}\right)\end{array}$ & $\begin{array}{r}7.65\left(10^{-3}\right) \\
\pm 1.50\left(10^{-2}\right)\end{array}$ & - \\
\hline
\end{tabular}

* The best-fit estimates shown with their corresponding uncertainties are derived from the approach to steady-state of the various solutes (washin phase, Fig. 2) and the absolute bile-to-plasma ratios observed at steady state. Parameters $r_{2}\left(0.3 \mathrm{~min}^{-1}\right), r_{3}\left(0.1 \mathrm{~min}^{-1}\right)$, and $\mathbf{r}_{7}\left(0.6 \mathrm{~min}^{-1}\right)$ were fixed in the final analyses, except where noted above. $¥$ Indicates insignificant contribution to transport of that solute; that is, not distinguishable from zero.

and horseradish peroxidase, and thus was not peculiar to dextran. This pattern was kinetically incompatible with simple movement between two compartments (perfusate and bile) via a pathway represented by a single rate constant. Instead, it required the presence of multiple, interposed compartments. The anatomical identity of these delay compartments is unknown; however, they would seem most likely to be intracellular. An alternative possibility of delay (poorly stirred) compartments in the paracellular pathway seems less likely from these studies alone for two reasons. First, it was not observed with either erythritol or sucrose, which presumably move at least in part via a paracellular pathway. Second, during the washout phase of the study, biliary dextran concentration exceeded that of perfusate for a time period much longer than that attributable to washout of the biliary deadspace $(5 \mathrm{~min}$ ) or to estimated time of diffusion through unstirred layers $(25$, 30). Third, the results of these nonsteady-state studies are

Table III. Percentage of Marker Recovered in 100,000 $\mathrm{g}$ Pellets in the Absence and Presence of Triton X-100*

\begin{tabular}{llr}
\hline Marker & $\begin{array}{l}\text { Marker in pellet } \\
\text { without Triton }\end{array}$ & \multicolumn{1}{l}{$\begin{array}{l}\text { Marker in pellet } \\
\text { with Triton }\end{array}$} \\
\hline & $\% \%$ & \multicolumn{1}{c}{$\%$} \\
Erythritol & $7(6.7-7.6)$ & $0.37(0.23-0.51)$ \\
Sucrose & $47(38-56)$ & $3.6(0.60-6.20)$ \\
Inulin & $35(28-42)$ & $0.56(0.52-0.60)$ \\
Dextran & $51(49-53)$ & $0.42(0.40-0.44)$
\end{tabular}

* In each experiment, the percentage of marker present in the $100,000 \mathrm{~g}$ pellet was determined in the absence and presence of $1 \%$ Triton X-100, both for the original supernatant (P2/H2 $\times 100, \mathrm{P}^{\prime} /$ $\mathrm{H} 2^{\prime} \times 100$, respectively) and for the rehomogenized pellet (P4/H4 $\times 100, \mathrm{P}^{\prime} / \mathrm{H}^{\prime} \times 100$, respectively). These two values agreed closely for all markers both in the presence and absence of detergent and are therefore combined in this table; ranges are indicated in parentheses. The numbers shown for each marker represent the mean values derived from two separate experiments. See Methods for details. analogous to those recently reported for sucrose and inulin movement in pancreas, an organ for which additional compelling evidence of a transcellular pathway exists (31).

Finally, using three different approaches, dextran, horseradish peroxidase, inulin, and sucrose were shown to be present

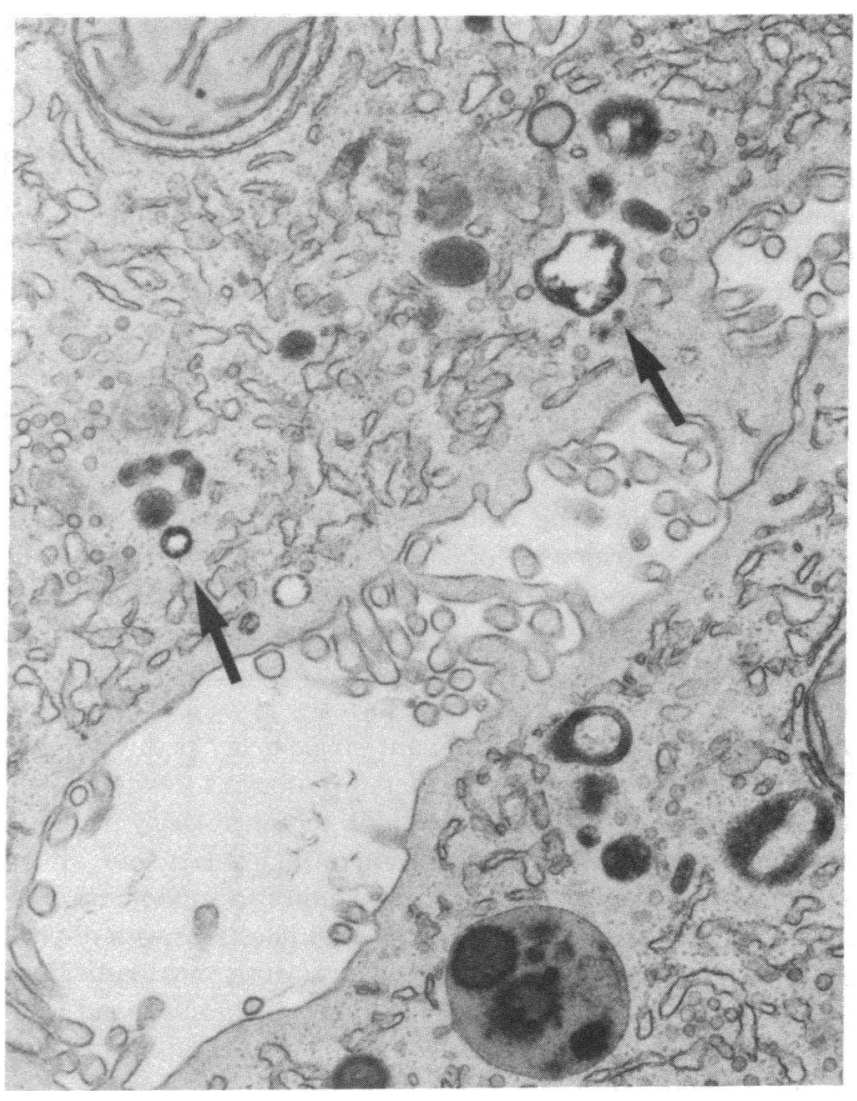

Figure 4. Electron micrograph of liver perfused for $60 \mathrm{~min}$ with perfusate containing $1 \mathrm{mg} / \mathrm{ml}$ of horseradish peroxidase demonstrating the presence of horseradish peroxidase in intracellular vesicles (arrows) $(\times 62,000)$. 

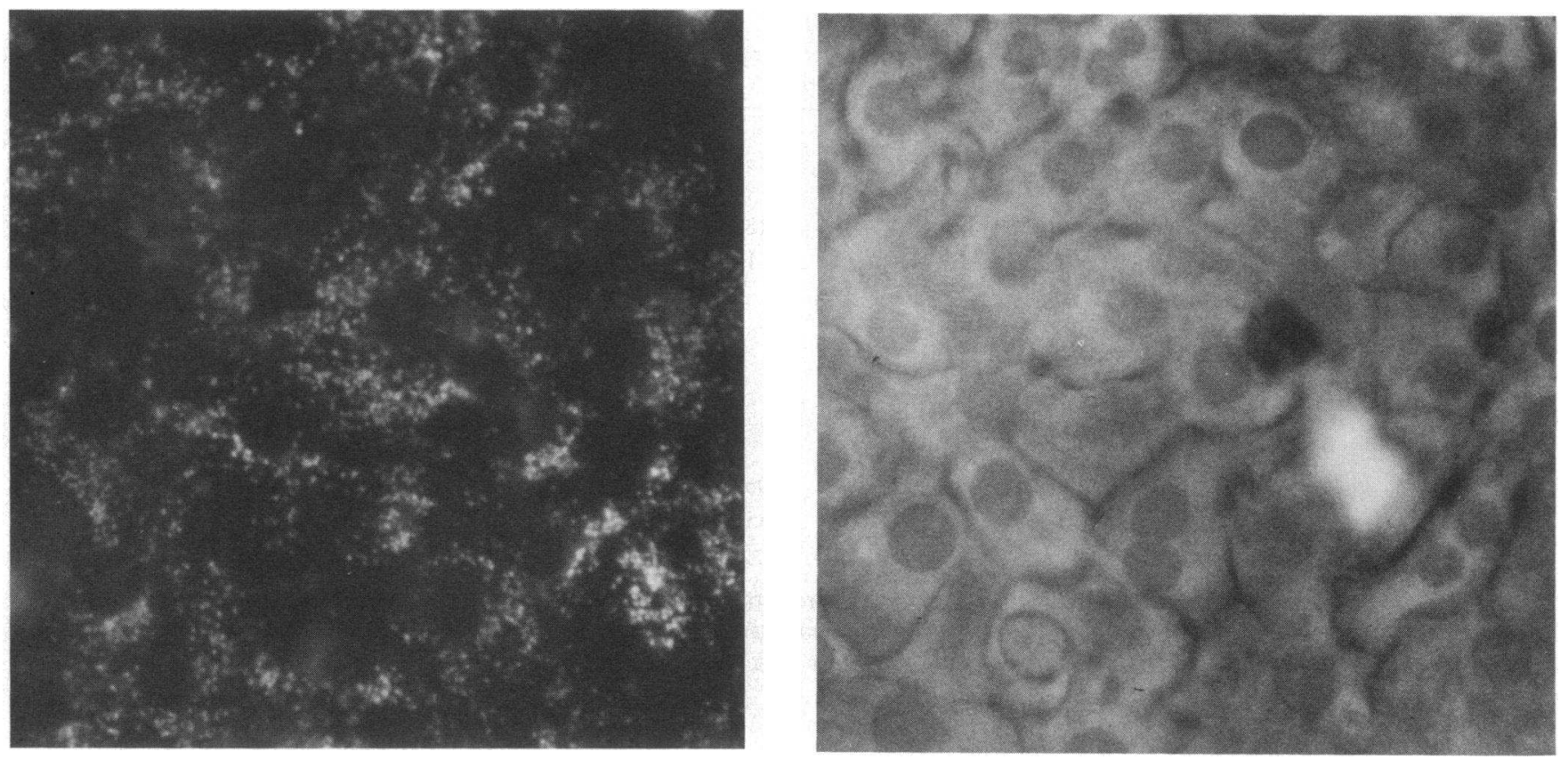

Figure 5. Fluorescent photomicrographs of cultured hepatocytes after 12-16 h incubation in medium containing fluorescein dextran (70,000 average mol wt) (left) and after a 10-min incubation in medium containing $45 \mu \mathrm{g} / \mathrm{ml}$ carboxyfluorescein diacetate (right).

in intracellular vesicles. For the reasons outlined in Results, the $35-51 \%$ association with intracellular vesicles (Table III) obtained in these studies probably represents a minimum estimate of the percentage of cell-associated isotope actually present in intracellular vesicles.

Collectively, these observations are most compatible with the participation of a transcytosis pathway in the blood-to-bile transport of inert markers. Based on the analyses of the nonsteady-state studies, it appears likely that the contribution of this pathway varies for different solutes. For erythritol, which is known to enter liver cells readily $(9 ; 10)$, and which exhibits exceedingly rapid (zero-delay) perfusate-to-bile movement that yields a calculated biliary deadspace less than the lowest conventional estimate based on taurocholate movement, a rapid transcellular convective pathway and/or paracellular pathway probably predominates (Fig. 3, Table II). These two possibilities cannot be distinguished based on the present results. The movement of dextran, microperoxidase, and horseradish peroxidase, by contrast, seems to be almost entirely dependent on transcytosis. The movement of sucrose, which is intermediate in size between microperoxidase and erythritol, appears to be dependent on transcytosis, as well as the zerodelay pathway and a predominant single-delay pathway (Fig. 3 , Table II). Because sucrose enters hepatocytes slowly $(9,10)$ (perhaps via endocytosis of bulk fluid), the single-delay pathway may represent paracellular movement. However, no direct evidence is provided by the present studies to support this.

These findings have readily evident implications with respect to canalicular bile formation. First, transcytosis may participate in the transport of a wide variety of macromolecules and may help explain the presence in bile of low concentrations of most serum proteins (32). Second, while a ouabain-insensitive, cytochalasin-sensitive mechanism for hepatocyte volume reguIation has been reported and attributed to canalicular-directed vesicles (33), the movement of bulk fluid via vesicles has been regarded generally as unimportant in secretion by liver or other tissues (34). The present findings suggest that the possible contribution of such a mechanism to bulk fluid movement by liver merits reexamination. Third, conclusions regarding canalicular permeability and/or tight junction integrity based on changes in the bile-to-plasma ratio of solutes such as sucrose after experimental manipulations, are clearly hazardous. For example, an agent that inhibits active solute transport and osmotically obligated water flux but has little effect on vesicular movement might be expected to result in an increase in biliary marker concentration independent of any changes in effective canalicular pore size or junctional integrity. This would pertain particularly to markers transported to bile wholly (e.g., dextran) or in part (sucrose) via a vesicular mechanism. The results for dextran are particularly instructive in this regard. Despite the results of nonsteady-state studies and tissue fractionation/cell visualization experiments that strongly implicate a vesicular pathway, it was possible to describe a plot of clearance versus bile flow (data not shown) under steady-state conditions by the conventional equation based on pore-restricted diffusion and bulk flow $(10,12)$. This observation points out the importance of nonsteady-state analyses and underscores the inadequacy of conformance to the conventional equation as a continued justification for the current paradigm that transport occurs solely via diffusion and filtration.

It is also important to explicitly acknowledge areas of persisting uncertainty as related to the present work. First, our studies permit no conclusions regarding the type of vesicles involved in marker secretion or the intracellular route taken. It is of interest in this regard that the biliary secretion of microperoxidase or horseradish peroxidase, which was measured by bioassay, was less than the secretion of the even larger molecular-weight compound, dextran, as measured by radiolabel. One potential explanation for this finding would be loss of enzymatic activity due to transit in part via a lysosomal 
pathway (35) or to encounter with an acidic environment, as reported by us and others for clathrin-coated vesicles and endosomes (36-38). Further speculation is not warranted based on the findings presented and will require additional study. Second, while selectivity based on molecular weight was not observed, it remains unclear whether selectivity based on other important parameters such as shape or charge might exist. We did not observe, for example, a difference in secretion of carboxyl (anionic) versus methoxy (neutral) inulin (data not shown), but selectivity for neutral as opposed to anionic solutes of similar size has been reported (29). It is interesting in this regard that morphologic studies using cationic ferritin have demonstrated selective enrichment of anionic sites in coated vesicles of vascular endothelium as compared with the rest of the plasma membrane (39). Thus, a vesicular pathway might exert charge selectivity. Third, while the present studies provide quantitative estimates of the contribution of transcytosis to the blood-to-bile transport of fluid phase markers, the implications with respect to bulk fluid movement via transcytosis are uncertain. Loss or accumulation of fluid by endocytic vesicles after their internalization would not be accompanied by corresponding changes in marker content, but only by reciprocal changes in marker concentration. Thus, biliary secretion of a fluid phase marker may not accurately reflect transcellular bulk fluid movement. Finally, biliary secretion of fluid phase marker will not provide a measure of the total amount of fluid internalized by hepatocytes via endocytosis, much of which may be recycled back to the space of Disse. At present, it seems that the perfusate-to-bile transport of markers such as dextran would provide only a minimum estimate $(\sim 0.1 \mu \mathrm{l} / \mathrm{g} \cdot \mathrm{min})$ of total fluid internalization via vesicles at the sinusoidal cell surface.

\section{Acknowledgments}

The authors wish to acknowledge the important contributions to the project of the University of California at San Francisco Liver Core Center-supported facilities (AM-26743) including the Perfused Rat Liver Facility (Dr. Richard Weisiger, director), the Cell Culture Facility (Dr. D. Montgomery Bissell, director), the Electron Microscopy Facility (Dr. Albert L. Jones, director), and the Biomathematics Facility (Dr. Vojtech Licko, director). The authors also wish to thank Jocelyn Matsumoto-Pon, Michael Wong, and Paul George for their technical support with selected aspects of these studies, Linda Kendrick for her expert help with the liver perfusions, and Diana Fedorchak and Michael Karasik for preparation of the manuscript and expert editorial assistance. Finally, the authors wish to thank Dr. Chin-Tzu Peng, Professor of Chemistry and Pharmaceutical Chemistry at the University of California at San Francisco, who prepared the radiolabeled dextran $(40,000 \mathrm{~mol} \mathrm{wt})$ used in these studies.

This work was supported in part by National Institutes of Health grants AM-26270, AM-26743, AM-07453, AM-01254, as well as grants from the Giannini Foundation, the American Liver Foundation, and The Walter C. Pew Fund for Gastrointestinal Research.

\section{References}

1. Ashwell, G., and J. Harford. 1982. Carbohydrate-specific receptors of the liver. Annu. Rev. Biochem. 51:531-554.

2. Goldstein, J. L., R. G. W. Anderson, and M. S. Brown. 1979. Coated pits, coated vesicles, and receptor-mediated endocytosis. Nature (Lond.). 279:679-685.
3. Renston, R. H., A. L. Jones, W. D. Christiansen, and G. T. Hradek. 1980. Evidence for a vesicular transport mechanism in hepatocytes for biliary secretion of immunoglobulin A. Science (Wash. DC). 208:1276-1278.

4. Schiff, J. M., M. M. Fisher, and B. J. Underdown. 1984. Receptor-mediated biliary transport of immunoglobulin A and asialoglycoprotein: sorting and missorting of ligands revealed by two radiolabeling methods. J. Cell Biol. 98:79-89.

5. Kacich, R. L., R. H. Renston, and A. L. Jones. 1983. Effects of cytochalasin $\mathrm{D}$ and colchicine on the uptake, translocation, and biliary secretion of horseradish peroxidase and $\left[{ }^{14} \mathrm{C}\right]$ taurocholate in the rat. Gastroenterology. 85:385-394.

6. Scharschmidt, B. F., and R. W. Van Dyke. 1983. Mechanisms of hepatic electrolyte transport. Gastroenterology. 85:1199-1214.

7. Smith, N. D., and J. L. Boyer. 1982. Permeability characteristics of bile duct in the rat. Am. J. Physiol. 242:G52-G57.

8. Barnhart, J. L., and B. Combes. 1978. Erythritol and mannitol clearances with taurocholate and secretin-induced choleresis. Am. J. Physiol. 234:E146-E156.

9. Forker, E. L. 1967. Two sites of bile formation as determined by mannitol and erythritol clearance in the guinea pig. J. Clin. Invest. 46:1189-1195.

10. Forker, E. L. 1968. Bile formation in guinea pigs: analysis with inert solutes of graded molecular radius. Am. J. Physiol. 215:56-62.

11. Renkin, E. M. 1954. Filtration, diffusion and molecular sieving through porous membranes. J. Gen. Physiol. 38:225-243.

12. Strasberg, S. M., C. N. Petrunka, R. G. Ilson, and J. E. Paloheimo. 1979. Characteristics of inert solute clearance by the monkey liver. Gastroenterology. 67:259-266.

13. Reichen, J., and M. Le. 1983. Taurocholate, but not taurodeoxycholate, increases biliary permeability to sucrose. Am. J. Physiol. 245:G651-G655.

14. Reichen, J., and M. Le. 1983. Effect of taurocholate on place and rate of entry of sucrose into the bile canaliculus. Gastroenterology 84:1283a. (Abstr.)

15. Elias, E., Z. Hruban, J. B. Wade, and J. L. Boyer. 1980. Phalloidin-induced cholestasis: a microfilament-mediated change in junctional complex permeability. Proc. Natl. Acad. Sci. USA. 77:22292233.

16. Enzymes and Related Biochemicals. 1978. Worthington Manual. Life Sciences Division, Worthington Biochemical, Freehold, NJ. 145146.

17. Van Dyke, R. W., J. E. Stephens, and B. F. Scharschmidt. 1982. Effect of ion substitution on bile acid-dependent and bile acidindependent bile formation by the isolated perfused rat liver. J. Clin. Invest. 70:505-517.

18. Van Dyke, R. W., J. L. Gollan, and B. F. Scharschmidt. 1983. Oxygen consumption by rat liver: effects of taurocholate and sulfobromophthalein transport, glucagon, and cation substitution. Am. J. Physiol. 244:G523-G531.

19. Scharschmidt, B. F., R. W. Van Dyke, and J. E. Stephens. 1982. Chloride transport by intact rat liver and cultured rat hepatocytes. Am. J. Physiol. 242:G628-G633.

20. Myers, B. D., F. Chui, M. Hilberman, and A. S. Michaels. 1979. Transtubular leakage of glomerular filtrate in human acute renal failure. Am. J. Physiol. 237:F319-F325.

21. Granath, K., and B. E. Kvist. 1967. Molecular weight distribution analysis by gel chromatography on Sephadex. J. Chromatogr. 28:6981.

22. Graham, K., and M. Karnovsky. 1966. The early stages of injected horseradishperoxidase in the proximal tubular cells of mouse kidney: ultrastructural cytochemistry by a new technique. J. Histochem. Cytochem. 14:291-302.

23. Bissell, D. M., L. E. Hammaker, and U. A. Meyer. 1973. Parenchymal cells from adult rat liver in nonproliferating monolayer culture. I. Functional studies. J. Cell. Biol. 59:722-734.

24. Scharschmidt, B. F., and J. E. Stephens. 1981. Sodium, chloride 
and taurocholate transport by cultured rat hepatocytes. Proc. Natl. Acad. Sci. USA. 78:986-990.

25. Hacki, W., and G. Paumgartner. 1973. Determination of biliary dead space using 14C-taurocholate as a marker. Experientia (Basel). 29:1091-1093.

26. Barber-Riley, G. 1963. Measurement of the capacity of the biliary tree. In The Biliary System. W. Taylor, editor. Blackwell Scientific Publications, Inc., Boston, MA. 89-103.

27. Barth, C. A., and L. R. Schwarz. 1982. Transcellular transport of fluorescein in hepatocyte monolayers: evidence for functional polarity of cells in culture. Proc. Natl. Acad. Sci. USA. 79:4985-4987.

28. Lorenzini, P., P. Ilter, P. Meier, and J. L. Boyer. 1982. Taurochenodeoxycholic acid stimulates hepatic uptake of $3 \mathrm{H}$-methoxyinulin in (3MI) into membrane-bound compartments. Hepatology (Baltimore). 2:737a. (Abstr.)

29. Bradley, S. E., and R. Merz. 1978. Permselectivity of biliary canalicular membrane in rats: clearance probe analysis. Am. J. Physiol. 235:E570-E576.

30. Tipping, E., and B. Ketterer. 1981. The influence of soluble binding proteins on lipophile transport and metabolism in hepatocytes. Biochem. J. 195:441-452.

31. Melese, T., and S. Rothman. 1983. Pancreatic epithelium is permeable to sucrose and inulin across secretory cells. Proc. Natl. Acad. Sci. U.S.A. 80:4870-4874.
32. Mullock, B. M., M. Dobrata, and R. H. Hinton. 1978. Sources of the proteins of rat bile. Biochim. Biophys. Acta. 543:497-507.

33. van Rossum, G. D. V., and M. A. Russo. 1981. Ouabainresistant mechanisms of volume control and the ultrastructural organization of liver slices recovering from swelling in vitro. J. Membr. Biol. 59:191-209.

34. Wheeler, H. O. 1975. Secretion of bile. In Diseases of the Liver. L. Schiff, editor. J. B. Lippincott Co., Philadelphia, PA. 87-110.

35. La Russo, N. F., and S. Fowler. 1979. Coordinate secretion of acid hydrolases into bile. J. Clin. Invest. 64:948-954.

36. Galloway, C. J., G. E. Dean, M. Marsh, G. Rudnick, and I. Mellman. 1983. Acidification of macrophage and fibroblast endocytic vesicles in vitro. Proc. Natl. Acad. Sci. USA. 80:3334-3338.

37. Forgac, M., L. Cantley, B. Wiedenmann, L. Altstiel, and D. Branton. 1983. Clathrin-coated vesicles contain an ATP-dependent proton pump. Proc. Natl. Acad. Sci. USA. 80:1300-1303.

38. Van Dyke, R. W., C. J. Steer, and B. F. Scharschmidt. 1984. Clathrin-coated vesicles from rat liver: enzymatic profile and characterization of ATP-dependent proton transport. Proc. Natl. Acad. Sci. USA. 81:3108-3112.

39. Simionescu, N., M. Simionescu, and G. E. Palade. 1981. Differentiated microdomains on the luminal surface of the capillary endothelium. I. Preferential distribution of anionic sites. J. Cell Biol. 90:605-613. 